\title{
Proteoglycans in articular cartilage revealed with a quick freezing and deep etching method
}

\author{
Hiroshi Toriumi, Hiroyuki Nakagawa, Hideho Ueda, Chong-Guang Leng, Yasuhisa Fujii, \\ Shinichi Ohno
}

\begin{abstract}
Objectives-To clarify the three dimensional ultrastructure of proteoglycans, and their relationship with other matrix components in articular cartilage.

Methods-Specimens from rat femoral heads were examined using three techniques: (1) Histochemical staining with cationic polyethyleneimine (PEI), using a pre-embedding or a postembedding method. Some tissues were pretreated with chondroitinase ABC or hyaluronidase. (2) Quick freezing and deep etching (QF-DE). Some specimens were fixed with paraformaldehyde and washed in buffer solution before quick freezing; others were frozen directly. (3) Ultrathin sections were studied after conventional preparation.
\end{abstract}

Results-Proteoglycans were observed as aggregated clumps with PEI staining by the pre-embedding method, but as fine filaments by the postembedding method. They were lost with enzyme digestion; this was also demonstrated by the QF-DE method. The ultrastructure was well preserved by the QF-DE method when fixation and washing procedures were included, but not without these procedures. A fine mesh-like structure was connected to the cell membrane in the pericellular matrix. Filamentous structures suggestive of aggrecans were observed among collagen fibrils. They had side chains, approximately $50 \mathrm{~nm}$ in length, which branched from the central filaments at intervals of $10-20 \mathrm{~nm}$, and were occasionally linked to other structures. Many thin filaments were also attached to the collagen fibrils.

Conclusions-The QF-DE method incorporating paraformaldehyde fixation and buffer washing procedures revealed three dimensional, extended structures suggestive of proteoglycans.

(Ann Rheum Dis 1996; 55: 466-474)

Articular cartilage characteristically lacks vessels, lymphatics, and a nerve supply. In response to the mechanical stresses of joint movements, it produces interstitial fluid flow with electrolytes, nutrients, and synthetic or waste products in the cartilagenous matrix. Articular cartilage also has a remarkably low coefficient of friction and a high resistance to mechanical abrasion that cannot be achieved by any prosthesis. ${ }^{1}$ In joint disorders such as rheumatoid arthritis and osteoarthritis, the articular cartilage is ultimately destroyed, resulting in loss of those properties. It is therefore important to understand the physical status of articular cartilage in vivo, in order to analyse the mechanisms of these disorders.

The structural arrangement of proteoglycans in articular cartilage has been described as predicted by various biochemical methods. ${ }^{2}$ However, they may undergo a variety of morphological changes, particularly in the case of proteoglycan aggregates, which can contain large quantities of solvent and are assumed to be present in a compressed state in vivo. ${ }^{134}$ In addition, the proteoglycan in articular cartilage is difficult to visualise on conventional ultrathin sections because routine techniques of preparation tend to modify it. ${ }^{5}$ Consequently, other techniques such as conventional fixation with cationic dyes ${ }^{6-17}$ and freeze substitution $^{3}{ }^{18-21}$ are frequently used to conserve its natural ultrastructure. Effects from chemical treatments cannot be excluded, and so the native structure of proteoglycan and its relationship with other components of the extracellular matrix remain unclear. In the past, native joint surface structure was revealed by freezing and scanning electron microscopy or transmission electron microscopy of low temperature replicas. ${ }^{22-24}$ In the present study, we examined the extracellular matrix in the middle layer of articular cartilage in rat femoral heads using the quick freezing and deep etching (QF-DE) method, ${ }^{25-32}$ which is currently believed to have less treatment effect on the native tissues.

Materials and methods

Forty male Wistar strain rats, weighing about $300 \mathrm{~g}$, were used in the study. Animals were anaesthetised with ether, the femoral heads were resected, and the articular cartilages with associated subchondral bones were cut off using razor blades.

A cationic dye, polyethyleneimine (PEI), ${ }^{7-10} 31$ was used to visualise negatively charged proteoglycans by both pre-embedding and postembedding methods; digestion with chondroitinase $\mathrm{ABC}$ or hyaluronidase was used to establish their localisation. Some specimens were frozen directly for a quick freezing and deep etching (QF-DE) technique without any pretreatment. Others were treated with PEI and enzymes in the pre-embedding method before 
being processed for the QF-DE method. Some of the specimens were fixed with $2 \%$ paraformaldehyde and washed in buffer solution. ${ }^{29-31}$ For comparison, ultrathin sections were prepared by a conventional method of fixation.

PRE-EMBEDDING METHOD FOR HISTOCHEMICAL PEI STAINING

The tissue blocks were fixed with $2 \%$ paraformaldehyde in $0.1 \mathrm{~mol} / 1$ sodium cacodylate (SC) buffer at $4^{\circ} \mathrm{C}$ for one hour, and then washed with the SC buffer. They were treated with a solution of $1 \cdot 1 \%$ sodium chloride $(\mathrm{NaCl})$ and $0.5 \%$ PEI (mol wt 70 000) (Wako Pure Chemical Industries Ltd, Osaka, Japan) in distilled water, adjusted with hydrochloric acid ( $\mathrm{HCl})$ to $\mathrm{pH} 7 \cdot 4$, at $4^{\circ} \mathrm{C}$ for one hour, ${ }^{9} 10$ then washed in the SC buffer and contrasted with a mixture of $2 \%$ phosphotungstic acid and $0 \cdot 1 \%$ glutaraldehyde in SC buffer at $4^{\circ} \mathrm{C}$ for one hour. After the buffer washing, they were postfixed with $1 \%$ osmium tetroxide for one hour, washed with the SC buffer, dehydrated in a graded series of ethanol, and embedded in epoxy resin. Ultrathin sections were prepared and stained with uranyl acetate and lead citrate.

Some specimens fixed with $2 \%$ paraformaldehyde were then digested with $1 \mathrm{U} / \mathrm{ml}$ chondroitinase ABC (Sigma Chemical Co, St Louis, USA) in $0.25 \mathrm{~mol} / 1$ Tris buffer, containing $0.18 \mathrm{~mol} / / \mathrm{NaCl}$ and $0.05 \%$ bovine serum albumin, at $37^{\circ} \mathrm{C}$ for one hour, and washed with the SC buffer. After that, they were processed for the same PEI staining as described above. Other specimens that had been digested with chondroitinase $\mathrm{ABC}$ or hyaluronidase $50 \mathrm{U} / \mathrm{ml}$ in $0.02 \mathrm{~mol} / 1$ sodium chloride acetate (Sigma), or stained histochemically with PEI, or both digested and stained, were processed for the QF-DE method, as described below.

\section{POSTEMBEDDING METHOD WITH PEI}

\section{HISTOCHEMISTR}

The cartilage tissue blocks were fixed with a mixture of $2 \%$ paraformaldehyde and $0.05 \%$ glutaraldehyde in the SC buffer for one hour, and washed with the same buffer. They were dehydrated in a graded series of dimethylformamide, embedded in LR White, and polymerised under the ultraviolet beam at $-20^{\circ} \mathrm{C}$ for 24 hours. Ultrathin sections $(75 \mathrm{~nm}$ thick) were then treated with PEI solution at room temperature for 15 minutes, and washed with the SC buffer. They were contrasted in a mixture of $2 \%$ phosphotungstic acid and $0 \cdot 1 \%$ glutaraldehyde in the SC buffer for 15 minutes, then postfixed with $1 \%$ osmium tetroxide in the SC buffer for 15 minutes, washed with the same buffer, and then stained with uranyl acetate and lead citrate.

Some ultrathin sections were digested with the chondroitinase $\mathrm{ABC}$ solution at $37^{\circ} \mathrm{C}$ for one hour, as described above. Then they were processed for the same PEI staining procedures.

The same experiments were repeated using another PEI, of molecular weight 2000 (Aldrich Chemical Co, Milwaukee, USA).
QUICK FREEZING AND DEEP ETCHING METHOD Immediately after removal, the cartilage tissue blocks were fixed with $2 \%$ paraformaldehyde in $0.1 \mathrm{~mol} / \mathrm{l}$ phosphate buffer, $\mathrm{pH} 7 \cdot 4$, for 30 minutes and the cut tissue surface sliced with a razor blade to remove surface matrix proteins solubilised in the buffer, as described previously. ${ }^{29-31}$ They were then rinsed in $10 \%$ methanol diluted with distilled water to prevent ice crystal formation, and quickly frozen on a copper block cooled with liquid nitrogen under a JFD-RFA freezing machine (JEOL, Japan). The cut tissue surfaces were carefully freeze fractured with a scalpel in liquid nitrogen, as described previously. ${ }^{29-31}$ The frozen specimens were put into an Eiko FD-3AS freeze fracture device (Eiko Engineering Ltd, Ibaraki, Japan) and deeply etched under vacuum conditions of $10^{-7}-10^{-8} \mathrm{~mm} \mathrm{Hg}$ at a temperature of $-95^{\circ} \mathrm{C}$ for $20-30$ minutes. After the deep etching, the specimens were rotary shadowed with platinum at an angle of $30^{\circ}$ and then with carbon at an angle of $90^{\circ}$. A drop of $2 \%$ collodion in amyl acetate solution was put onto the replica membranes as soon as they were removed from the machine, in order to prevent them from breaking into pieces during the following digestion procedure. The replicas coated with dried collodion were treated in 0.1 $\mathrm{mol} / 1 \mathrm{HCl}$ solution and with household bleach for 30-60 minutes to dissolve the tissue components, and transferred into distilled water, from which they were placed on Formvar filmed copper grids and immersed in amyl acetate solution to dissolve the dried collodion.

Some fresh tissue blocks were frozen immediately after extirpation without any treatment and processed for the QF-DE method, as described above.

PREPARATION OF CONVENTIONAL ULTRATHIN SECTIONS

Some tissue blocks were routinely fixed with $2.5 \%$ glutaraldehyde in phosphate buffer for one hour and postfixed with $1 \%$ osmium tetroxide in phosphate buffer for one hour. They were dehydrated with a graded series of ethanol and embedded in epoxy resin. Ultrathin sections of the specimens were prepared, and stained with uranyl acetate and lead citrate.

All replica membranes and ultrathin sections were observed with transmission electron microscopes (Hitachi $\mathrm{H}-600$ and $\mathrm{H}-8100$ ) at an accelerating voltage of $75 \mathrm{kV}$. Some stereo pictures were taken at tilting angles of $\pm 5^{\circ}$. Electron micrographs of the replicas were printed from the inverted negative films in order to make platinum deposits appear white.

\section{Results}

CONVENTIONAL ULTRATHIN SECTION STUDY The cell surface was undulating, and with cell processes protruding from it. The pericellular matrix formed a clear zone in which electron density was relatively low; morphological structures were scarce, but numerous electron dense granules were observed. The sizes of the 
granules became smaller the further they were from the chondrocytes, and their electron density decreased similarly. Other granules were also observed between the collagen fibrils in the interterritorial matrix (fig 1).

HISTOCHEMICAL PEI STAINING

With the pre-embedding histochemical method, 'winged' granules were clearly observed throughout the extracellular matrix. ${ }^{9}{ }^{10}$ Those in the pericellular matrix were bigger than those in the interterritorial matrix. Some appeared to be connected to the cell membrane of the chondrocytes and to collagen fibrils (fig $2 \mathrm{~A}, \mathrm{D}$ ). In contrast, with the postembedding method no clear granules were observed, but fine filamentous structures could be seen in the pericellular matrix in particular (fig 3A); they were lost after chondroitinase $A B C$ digestion. In general, the destruction of tissues as a result of the enzyme treatment was revealed by the pre-embedding method but, except for the destruction of the fine filamentous structures, not by the postembedding technique (figs $2 \mathrm{~B}, \mathrm{E}, 3 \mathrm{~B}$ ).

In the replica specimens stained with PEI, the extracellular matrix was precipitated, and hollows were formed among the precipitated products. In the interterritorial matrix, the collagen fibril network was destroyed, and the filamentous structures were aggregated around

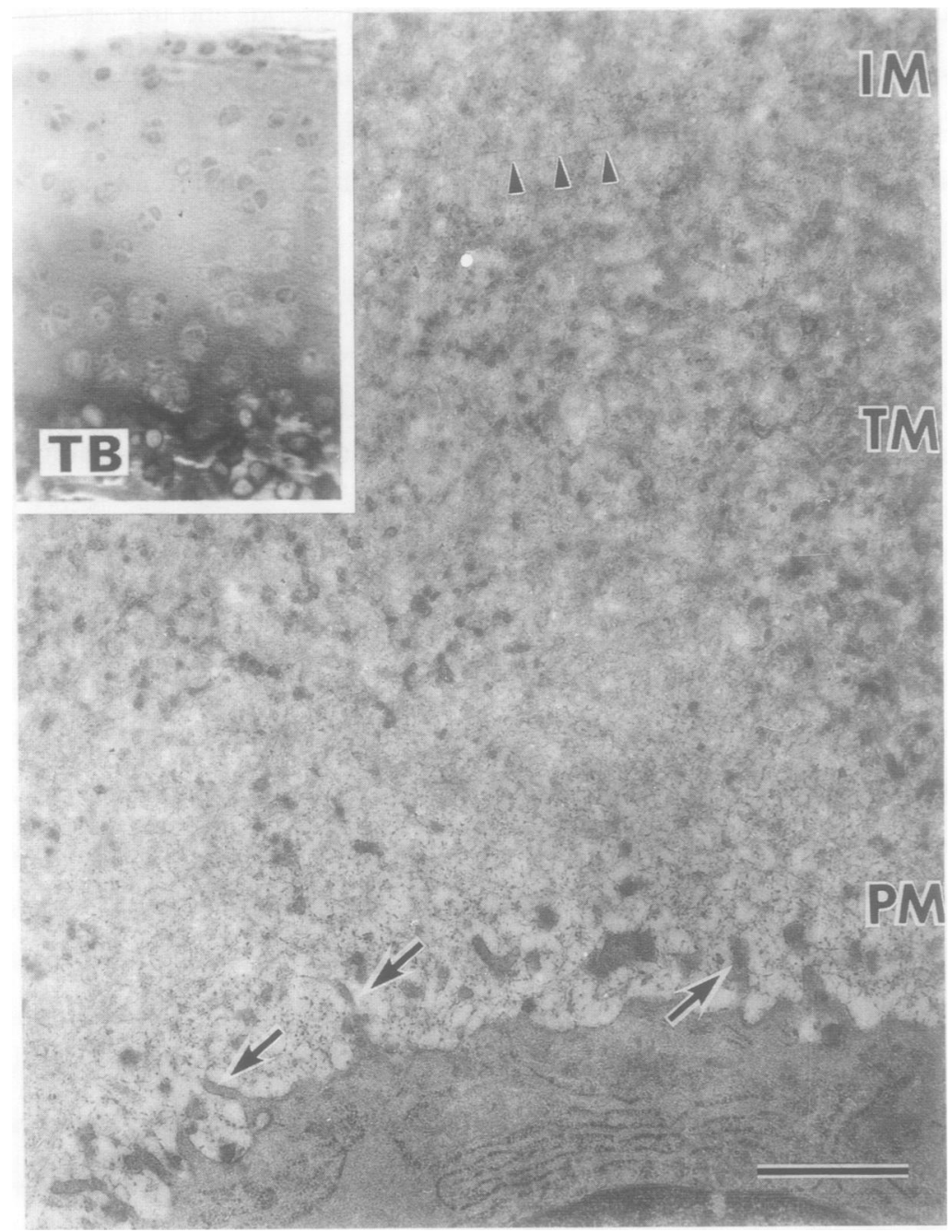

the collagen fibrils. They were also aggregated with substances suggestive of PEI molecules. In the replica specimens digested with the enzymes and treated with PEI, some filamentous structures disappeared and others formed rough aggregates around collagen fibrils (fig $2 \mathrm{C}, \mathrm{F}$ ).

The same findings were observed in the specimens treated with the PEI of molecular weight 2000 .

QUICK FREEZE-DEEP ETCHING STUDY

In the replica specimens that were frozen immediately after removal without any treatment, fine structures were rarely identified, even in the limited surface structures (fig 4A); they appeared, rather, to be buried in amorphous material. In the replica specimens that were fixed with paraformaldehyde and washed in the buffer solution before the freezing, fine, three dimensional extracellular matrical structures could be observed (fig 4B). The following findings relate mainly to the latter replica preparations.

Cytoplasmic processes and vesicular structures were observed along the smooth cell surface, and cell organelles were clearly visible in the cytoplasm (fig 5).

In the pericellular matrix, many filaments were closely attached to the cell membrane and processes of the chondrocyte, forming a meshwork. The sizes of the pores in the mesh increased gradually the further they were from the cell surface, and the filaments also became thicker in the same fashion. The meshwork was continuous, without any clear borders, across the pericellular area to the territorial and interterritorial matrices. Distinct fibrils ran parallel to the cell surface from the edge of the pericellular area to the territorial matrices; they were approximately $20 \mathrm{~nm}$ in diameter and laterally branched. The granular structures that were usually observed in conventional ultrathin sections were not found in these replica preparations (fig 5).

In the interterritorial matrix, thick collagen fibrils, varying from 30 to $110 \mathrm{~nm}$ in diameter, formed a three dimensional network, within which a large number of extended filaments were observed without precipitation. They were frequently straight and had side chains about $30-80 \mathrm{~nm}$ in length and at approximately 10-20 nm intervals; some were symmetrically branched more or less at right angles. In general they did not overlap each other; occasionally they were linked to other structures (figs 6,7 ). In the territorial matrix, collagen fibrils were more sparsely distributed

Figure 1 Electron micrograph of articular cartilage in a conventional ultrathin section. The cell membrane surface is undulating, and cell processes (arrows) protrude from the surface. The pericellular matrix (PM) forms a clear zone of low electron density, and gives way to the territorial matrix (TM), where collagen fibrils are thin. In the interterritorial matrix (IM), collagen fibrils are thick and have clear striations (arrowheads). Numerous electron dense granules are present, especially in the pericellular matrix. Bar represents 1 mm. Inset: Light micrograph $(\times 40)$ of articular cartilage in a thick section stained with toluidine blue. 

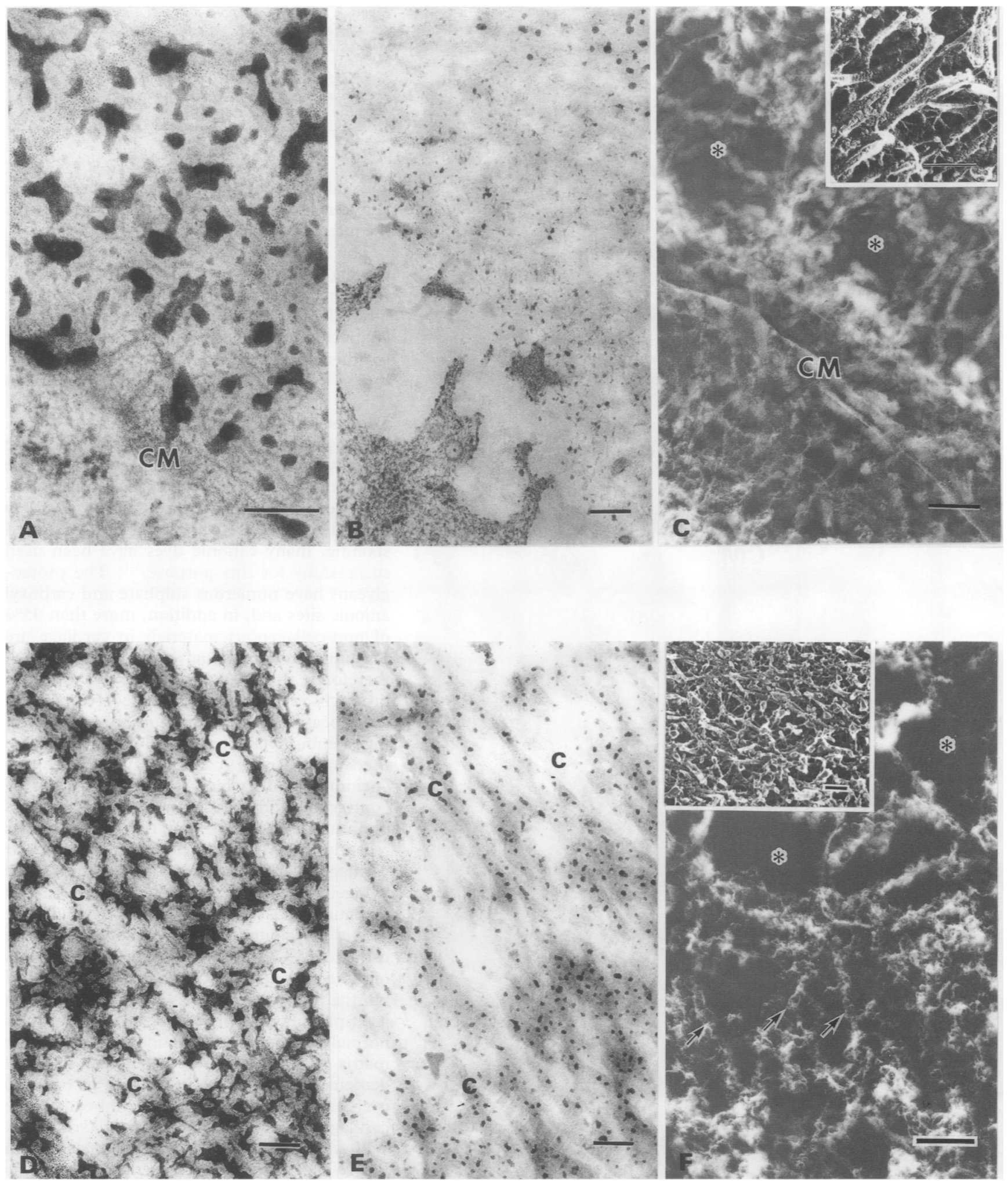

Figure 2 Histochemical polyethyleneimine (PEI) staining by the pre-embedding method. A-C: Pericellular matrix. D-F: Interterritorial matrix. $\boldsymbol{A}, \mathrm{D}$ : Ultrathin sections prepared after the PEI staining. Winged structures are precipitated states of proteoglycan and PEI: some are connected to the cell membrane (CM) and collagen fibrils $(C)$ in two dimensional views. $\boldsymbol{B}, \boldsymbol{E}$ : Ultrathin sections obtained from specimens digested with chondroitinase $A B C$, and then stained with PEI. Small electron dense granules, which are assumed to have survived the enzyme digestion, are present throughout the pericellular (B) or interterritorial (E) matrix, but the large winged structures have disappeared completely. $\boldsymbol{C}, \boldsymbol{F}:$ Replica electron micrographs prepared after the PEI staining. The fine structures are aggregated and indistinguishable. Electron lucent hollows (asterisks) are formed among precipitated products; among the latter are irregular structures covered with aggregated particles suggestive of PEI (arrows). Insets: Chondroitinase ABC (C), and hyaluronidase (F) treatments. Bar represents $200 \mathrm{~nm}$. 

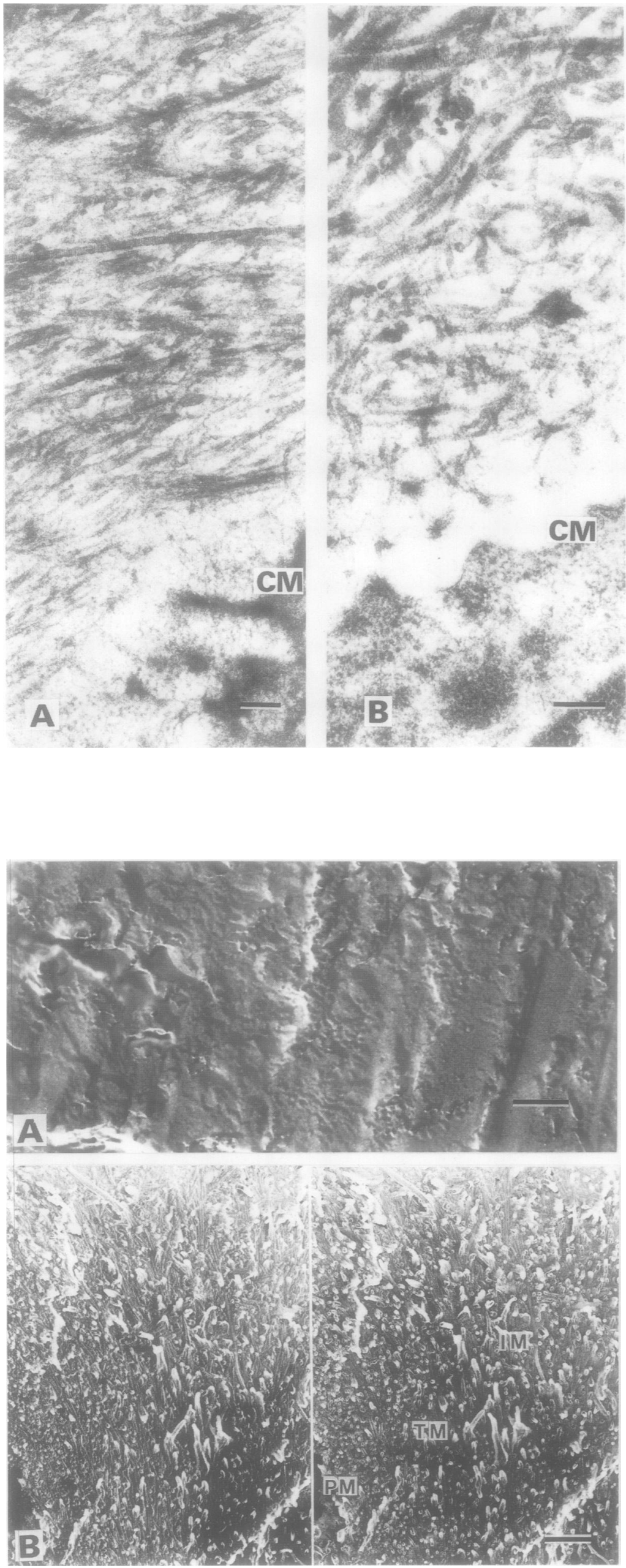

Figure 3 Ultrathin sections prepared after histochemical polyethyleneimine (PEI) staining by the postembedding method. Fine filamentous structures between collagen fibrils, especially in the pericellular matrix, some of which are connected directly to the cell membrane (CM) (A), disappeared completely after chondroitinase ABC digestion (B). Bar represents $200 \mathrm{~nm}$.

and thinner, and the filamentous structures between them more abundant, compared with the interterritorial matrix (fig 8).

The collagen fibrils had numerous and varying, thin and short (5-30 $\mathrm{nm}$ in length) filaments protruding from them at irregular intervals. Occasionally, these lateral filaments connected the collagen fibrils to each other or to other structures (figs 6,9 ).

\section{Discussion}

Proteoglycans are easily destroyed during the processes of preparation of specimens for morphological studies, and considerable efforts have been made to visualise them in a variety of tissues. Since Luft first used ruthenium red staining, many cationic dyes have been used successfully for this purpose. ${ }^{6-17}$ The proteoglycans have numerous sulphate and carboxyl anionic sites and, in addition, more than $95 \%$ of non-collagenous materials in cartilage are presumed to be proteoglycans. ${ }^{33}$ It is believed, therefore, that most of the dye reactive products in cartilage are proteoglycans, and their location and distribution are revealed by the staining characteristics. In this study, we used one of the cationic dyes, polyethyleneimine (PEI), which is easily visualised by contrasting with heavy metals such as phosphotungstic acid or osmium tetroxide, ${ }^{7}$ and has recently been applied to staining articular cartilage. ${ }^{7-10}$ We have been able to demonstrate 'winged' structures similar to those described by Sauren et al. ${ }^{9} 10$

The precipitation of proteoglycans with cationic dyes has been reported to prevent loss of proteoglycans from cartilaginous tissues, ${ }^{3}$ 6-17 but the term 'precipitation of proteoglycans' in itself probably implies the destruction of their normal structure and relationship with other matrix components-a phenomenon well demonstrated in figure $2 \mathrm{C}$ and $\mathrm{F}$. In order to overcome this problem, we embedded the cartilage specimens in the hydrophilic resin, LR White, and stained them histochemically with PEI by the postembedding method. In this way it was possible to observe, particularly in the pericellular matrix, fine filamentous structures which we consider to be abundant proteoglycans. Their disappearance after digestion

Figure 4 Replica electron micrographs without any treatment $(\boldsymbol{A})$ and with buffer washing $(\boldsymbol{B})$. A: Freeze fractured surface relatively flat, as if the structure is buried in amorphous material. No clear matrix structural components; only surface structures evident. Bar represents $200 \mathrm{~nm}$. B: Stereo pictures of the extracellular matrix prepared after paraformaldehyde fixation and buffer washing, clearly showing the fibrous structural organisation three dimensionally. The collagen fibrils become thicker towards the territorial periphery (TM). PM = Pericellular matrix; IM = interterritorial matrix. Bar represents $1 \mu \mathrm{m}$. 
with chondroitinase $\mathrm{ABC}$ also suggests that they were mostly proteoglycans. This postembedding histochemical method is considered useful for visualising native proteoglycans in an extended state without precipitation. However, proteoglycan profiles obtained with this method were very delicate. In addition, it was difficult to achieve a uniform infiltration of PEI particles and other contrasting agents throughout the entire section, and the PEI probably reacted with anionic sites of proteoglycans only on the surface of the ultrathin sections. Furthermore, it was difficult to clarify the relationship between proteoglycans and other structures that were superimposed in the sections. Even the quick freezing and freeze substitution method, which has been found useful for preserving native articular cartilage, ${ }^{318-21}$ may not resolve this problem because of its two dimensional perspective, and three dimensional analysis on the basis of two dimensional profiles thus remains problematic. ${ }^{34}$

With the quick freezing-deep etching method, and with buffer washing procedures performed after the paraformaldehyde fixation, fine extracellular structures could be visualised three dimensionally. In our metal contact method with liquid nitrogen, cartilaginous tissue blocks were quickly frozen without visible ice crystal formation within a depth of approximately $8 \mu \mathrm{m} \cdot{ }^{27-31} \mathrm{It}$ is probable that this cryofixation process is more natural than those using conventional chemical fixation, ${ }^{32}$ and that as a result, tissue structures may be observed in a relatively natural state. In living tissues, however, there are always many extracellular materials, including soluble proteins related to cellular metabolism; when these are not removed during preparation of the specimen, the extracellular structures remain obscured and unobservable. Thus in specimens that were not washed with buffer solution but were frozen immediately after their removal, extracellular matrix components were rarely displayed, but amorphous structures, under which the structural components were located, appeared to constitute the extracellular matrix. These must be removed in order to observe the matrix components; if not, the morphological profiles obtained represent not native extracellular matrix components, but modified ones. In the present study, we used the buffer washing method after paraformaldehyde fixation, and were able to visualise fine structures that we believe were representative of the insoluble extracellular matrix components, though they may have been modified to some extent by these procedures.

As shown in figure 5, a highly interconnected filamentous meshwork was observed in the peri-

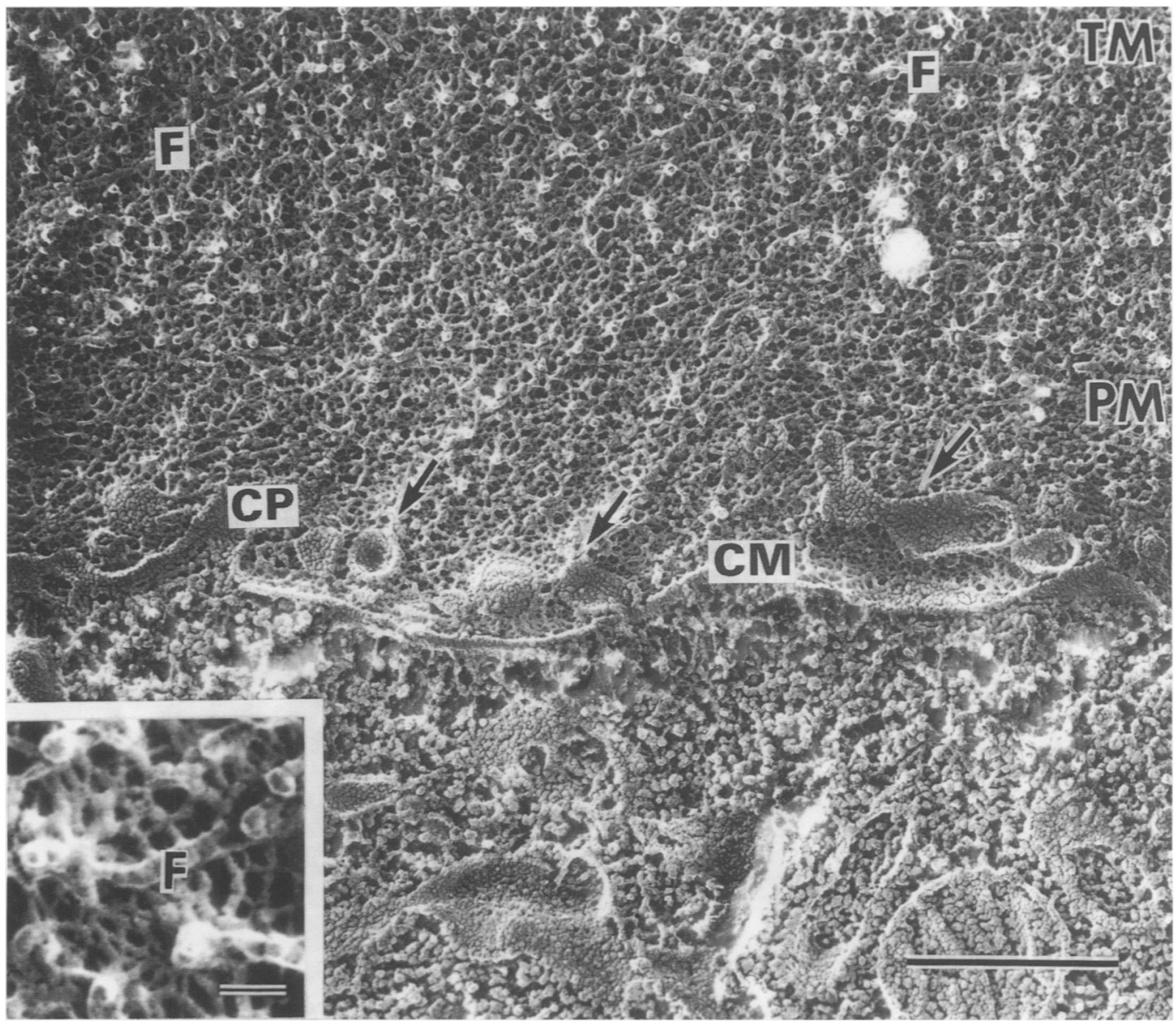

Figure 5 Replica electron micrographs of a chondrocyte and the pericellular matrix. The cell membrane (CM) surface is smoothly contoured, and cell processes (CP) may be observed. Horizontally freeze fractured processes or vesicular structures (arrows) are seen on the cell surface, and cell organelles are clearly visible in the cytoplasm. A filamentous structure is connected to the cell membrane, and runs from the pericellular matrix (PM) to the territorial matrix (TM) without a clear border. Distinct fibrils $(F)$ run parallel to the cell surface from the edge of the pericellular matrix to the territorial matrix. Bar represents $500 \mathrm{~nm}$. Inset: Laterally branched fibrils. Bar represents $50 \mathrm{~nm}$. 


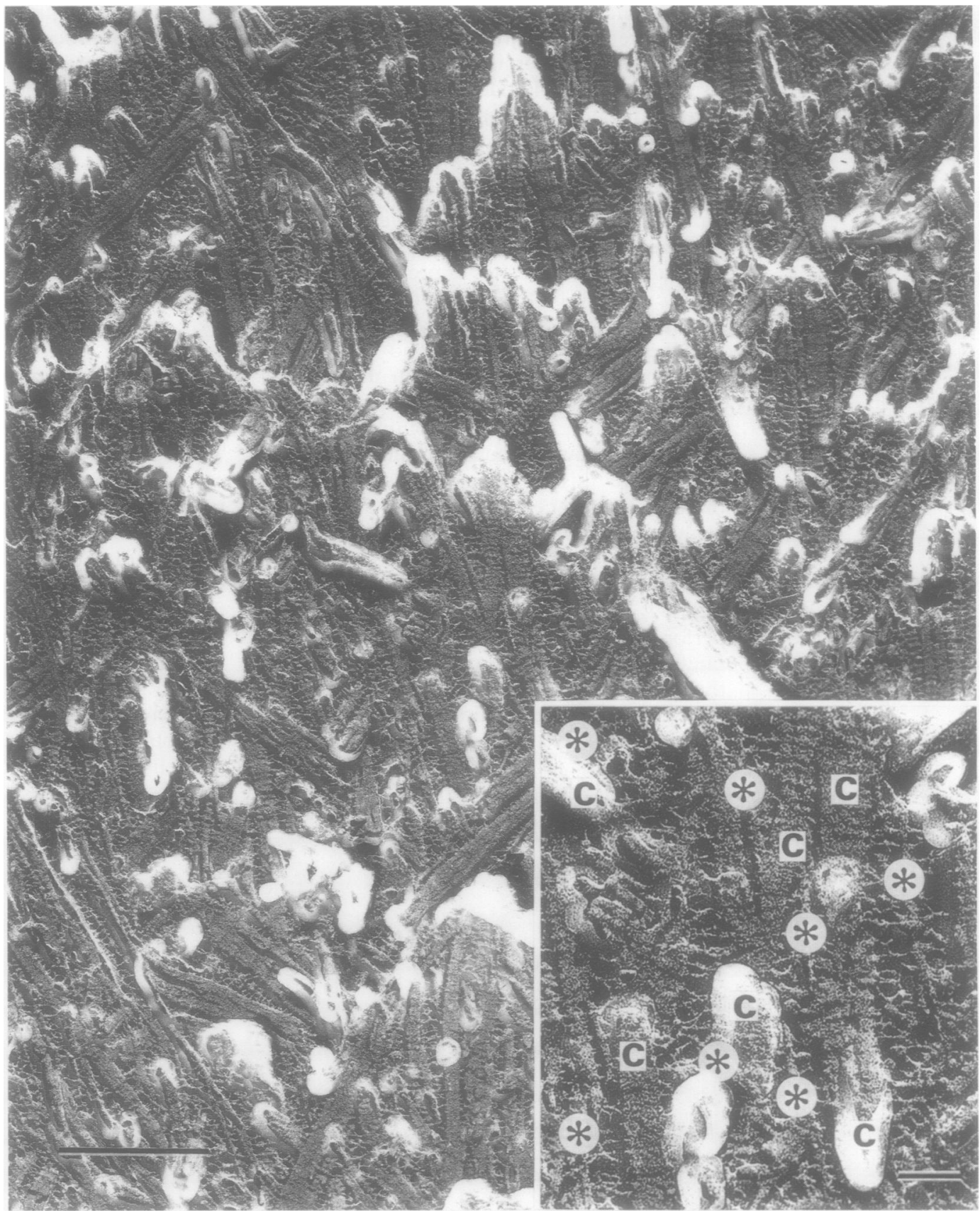

Figure 6 Interterritorial matrix. Thick collagen fibrils form three dimensional lattice structures in which bundles of fibres including relatively thin collagen fibrils run. Bar represents $500 \mathrm{~nm}$. Inset: Higher magnification of the middle area. Among the collagen fibrils (C), there are several filamentous structures (asterisks), which fill the intercollagenous spaces: they have laterally protruding side chains, some of which are connected to other structures. Bar represents $100 \mathrm{~nm}$.
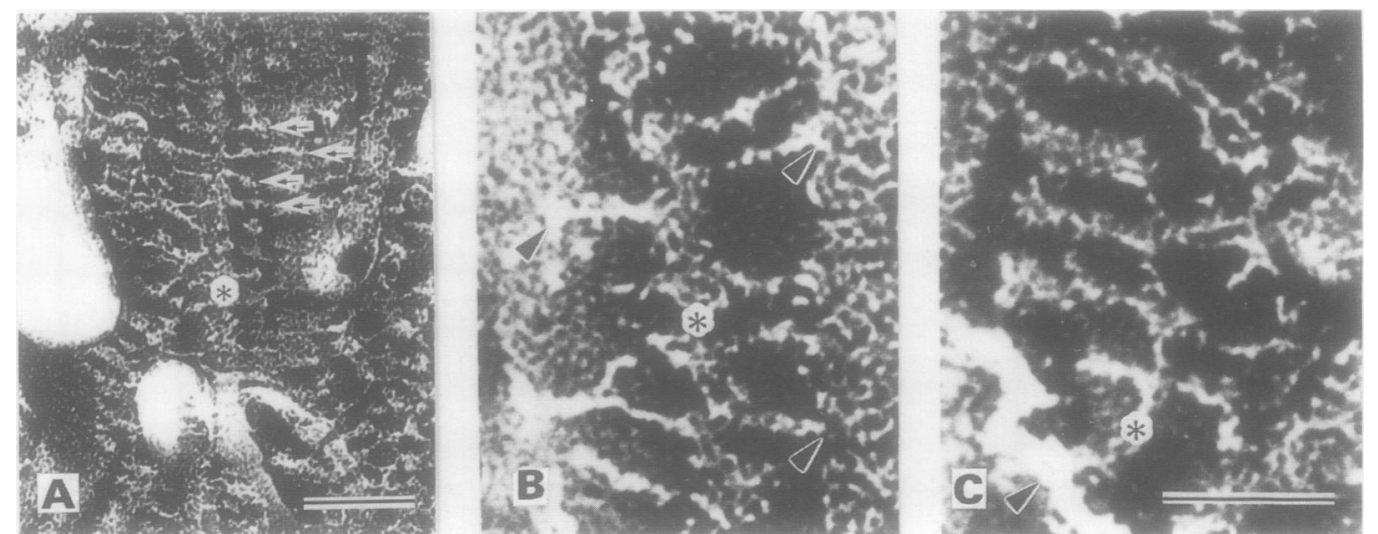

Figure 7 Structures suggestive of aggrecans, which were revealed by the quick freezing-deep etching method. They consist of central filaments (A, B: asterisks), which are frequently straight, usually with side chains branching off (some symmetrically (A: arrows)) at intervals of 10-20 nm. The side chains also link to collagen fibrils (B: arrowheads). Some central filaments seem to be interconnected to undulating fibres (C: arrowhead). A: Bar represents $100 \mathrm{~nm} ; \boldsymbol{B}, \boldsymbol{C}:$ bar represents $50 \mathrm{~nm}$. 


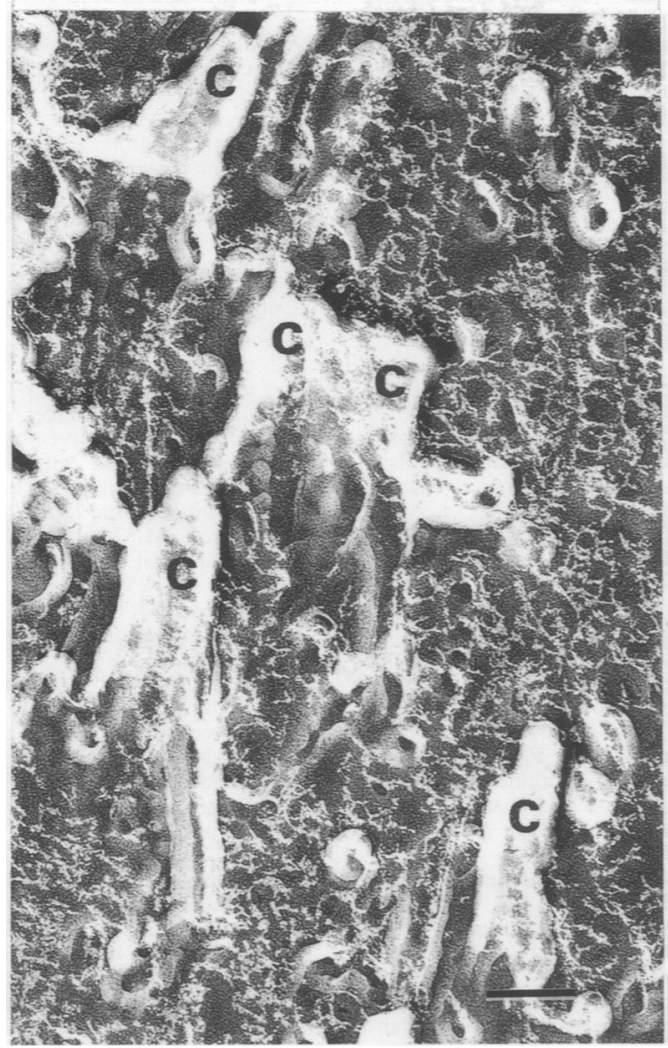

Figure 8 Territorial matrix. Collagen fibrils $(C)$ are more loosely distributed and thinner than those in the interterritorial matrix. Filamentous structures between the collagen fibrils appear abundant and their proportion is

larger than that in the interterritorial matrix. Bar represents $100 \mathrm{~nm}$.
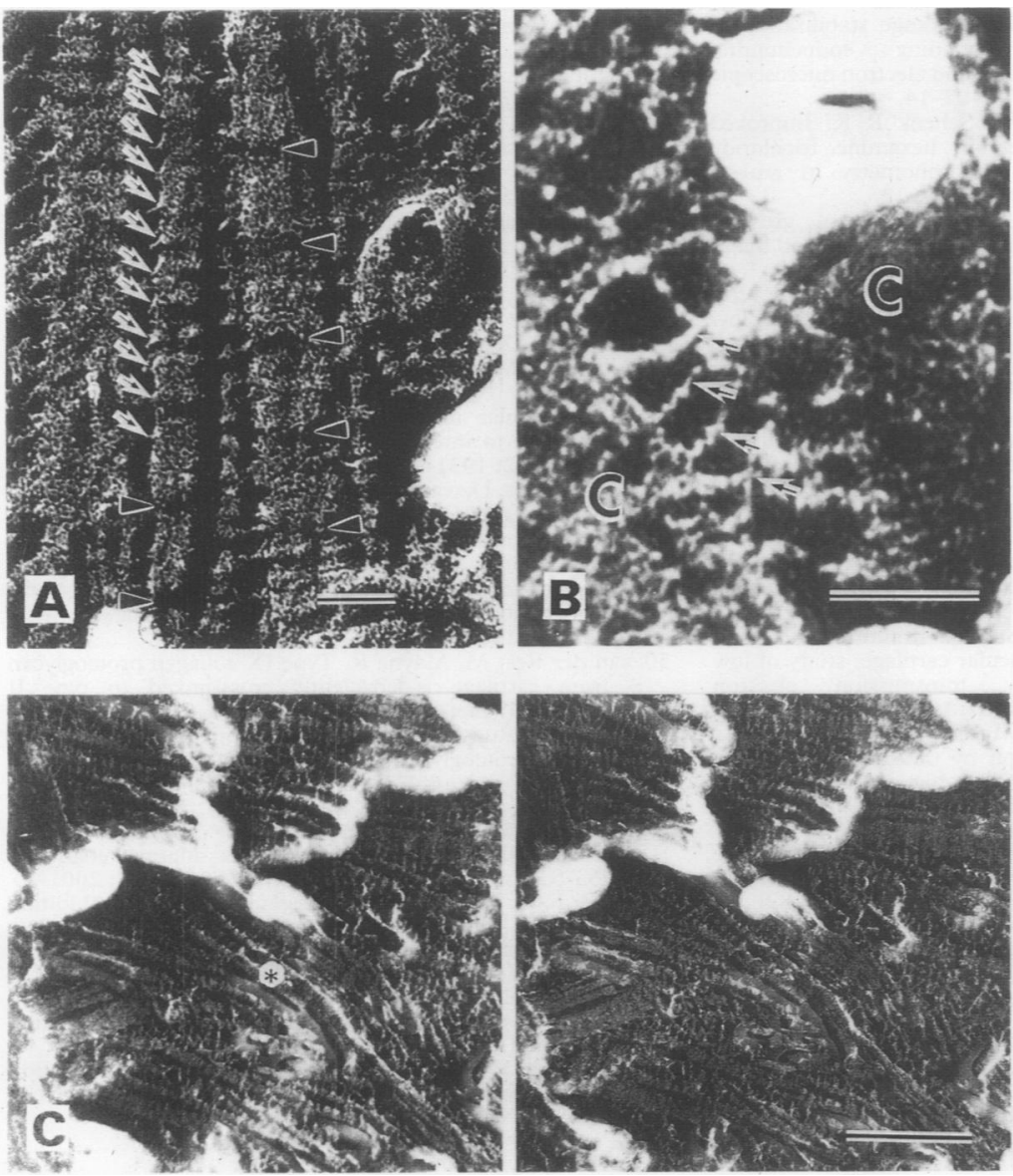

cellular matrix, and was closely connected to the cell membrane of a chondrocyte. Numerous filamentous structures with side chains, appearing as winged structures in the specimens prepared by the PEI pre-embedding method, were found among the networks of collagen fibrils in the territorial and interterritorial matrices and we suggest them to be proteoglycans-specifically, aggrecans. Their side chains suggestive of glycosaminoglycans were approximately $50 \mathrm{~nm}$ in length, and branched off from the central filaments suggestive of core proteins at intervals of $10-20 \mathrm{~nm}$. Previously reported sizes and structures of aggrecans were based on findings from two dimensional observations on extracted specimens: ${ }^{635-44}$ reported lengths of chondroitin sulphate chains ranged from 38.6 to $73 \mathrm{~nm},{ }^{353742} 44$ and the branching intervals were 7 to $13.9 \mathrm{~nm} .^{6353744}$ Using the quick freezing and freeze substitution method, their lengths have been estimated as $40-60 \mathrm{~nm}$ and the intervals as $5-15 \mathrm{~nm} ;{ }^{19}$ the length has also been reported to be almost $40 \mathrm{~nm} .{ }^{45}$ Our data show close agreement with these. Proteoglycan aggregates are in a hydrated state and compacted in the native tissue, and their swelling pressure is the source of viscoelasticity of the articular cartilage. ${ }^{1}$ Wight et al reported that they were compressed by the collagen fibril network to one fifth of their normal size; ${ }^{4}$ Hunzicker et al reported values of $1 / 3 \cdot 5-1 / 4 .^{3} \mathrm{It}$ is suggested that compaction of the aggregates is the result, not of a reduction in the size of the aggrecans, but of an increase in their number.

We found that many kinds of thin filaments measuring 5-30 $\mathrm{nm}$ protruded from the collagen fibrils, and occasionally connected them to other structures. Heterogeneous collagen fibrils of the articular cartilage have been shown to be composed of type II, IX, and XI collagens. ${ }^{46}$ The type IX collagens have been reported to be distributed on the surface of the fibrils. ${ }^{47-50} \mathrm{Small}$ proteoglycans, such as decorin and fibromodulin, are also reported to be scattered on the collagen fibril surface, ${ }^{51} 52$ and regulate the formation of thick collagen fibrils and networks, ${ }^{47495354}$ and cytokine activity. ${ }^{55}$ Further studies are required to determine the nature of the components represented by these filamentous structures.

Figure 9 Replica electron micrographs of small filaments attached to the collagen fibrils and surface structures on the fibrils, revealed by the quick freezing-deep etching method. A: Striations (arrowheads) in thick collagen fibrils, but not in the thin fibrils. The collagen fibrils also have pleated surface structures (arrows), that protrude with an almost regular periodicity. Bar represents $100 \mathrm{~nm}$. B: Helical filaments (arrows) are observed in some parts, where

collagen fibrils intersect three dimensionally. Bar represents $50 \mathrm{~nm}$. C: Stereo pictures of fibrils fusing (asterisk) into a thicker collagen fibril. Numerous thin and short filaments are also evident three dimensionally on the collagen fibrils. Bar represents $300 \mathrm{~nm}$. 
1 Mow V, Rosenwasser M. Articular cartilage: biomechanics. In: Woo S L Y, Buckwalter J A, eds. Injury and repair of the musculoskeletal soft tissues. Park Ridge, IL: American Academy of Orthopedic Surgeons, 1991; 427-63.

2 Doege K J, Sasaki M, Kimura T, Yamada Y. Complete coding sequence and deduced primary structure of human cartilage large aggregating proteoglycans, aggrecan. Human-specific repeats, and additiona alternating spliced forms. I Biol Chem 1991; 266: 894-902.

3 Hunzicker E B, Schenk R K. Structural organization of proteoglycans in cartilage. In: Wight $\mathrm{T}$, Mecham R, eds. Biology of proteoglycans. New York: Academic Press, 1987; $155-85$.

4 Wight T I:, Heinegàrd D K, Hascall V C. Proteoglycans: structure and function. In: Hay E D, ed. Cell biology of extracellular matrix, 2nd edn. New York: Plenum Press, $1991 ; 45-78$.

5 Engfeldt B, Hjertquist S-O. Study on the epiphysial growth zone. 1. The preservation of acid glycosaminoglycans in tissues in some histotechnical procedures for electron microscopy. Virchows Arch B 1968; 1: 222-9.

6 Thyberg J. Electron microscopy of cartilage proteoglycans. Histochem f 1977; 9: 259-66.

7 Schurer J W, Hoedemaeker P J, Molenaar I. Polyethyleneimine as tracer particle for (immuno) electron microscopy. F Histochem Cytochem 1977; 25: 384-7.

8 Schurer J W, Kalicharan D, Hoedemaeker P J, Molenaar I. The use of polyethyleneimine for demonstration of The use of polyethyleneimine for demonstration of
anionic sites in basement membranes and collagen fibrils. J Histochem Cytochem 1978; 26: 688-9.

9 Sauren Y M H F, Mieremet R H P, Groot C G Koerten H K, Scherft J P. Polyethyleneimine as a contras agent for ultrastructural localization and characterization of proteoglycans in the matrix of cartilage and bone. $\mathscr{F}$ Histochem Cytochem 1991; 39: 331-40.

10 Sauren Y M H F, Mieremet R H P, Lafeber F P J G, Huber-Bruning O, Groot C G, Scherft J P. Changes in proteoglycans of aging and osteoarthritic human articular cartilage: an electron microscopic study with polyethyleneimine. Anat Rec 1994; 240: 208-16.

11 Luft J H. Ruthenium red and violet. 1. Chemistry purification, methods of use for electron microscopy and mechanism of action. Anat Rec 1971; 171: 347-68.

12 Luft J H. Ruthenium red and violet. II. Fine structura localization in animal tissues. Anat $\operatorname{Rec} 1971 ; 171$ $369-416$

13 Ruggeri A, Dell'Orbo C, Quacci D. Electron microscopic visualization of proteoglycans with alcian blue. Histochem f 1975; 7: 187-97.

14 Shepard N, Mitchell N. Simultaneous localization of proteoglycan by light and electron microscopy using toluidine blue $\mathrm{O}$. A study of epiphyseal cartilage. $\mathscr{f}$ Histochem Cytochem 1976; 24: 621-9.

15 Scott J E. Collagen-proteoglycan interactions. Localization of proteoglycans in tendon by electron microscopy: of proteoglycans in tendon
Biochem f 1980; 187: 887-91.

16 Shepard N, Mitchell N. Acridine orange stabilization of glycosaminoglycans in beginning endochondral ossification. A comparative light and electron microscopic study. Histochemistry 1981; 70: 107-14.

17 Hunzicker E B, Herrmann W, Schenk R K. Improved cartilage fixation by ruthenium hexamine trichloride (RHT). A prerequisite for morphometry in growth cartilage. 7 Ultrastruct Res 1982; 81: 1-12.

18 Akisaka T, Shigenaga Y. Ultrastructure of growing epiphyseal cartilage processed by rapid freezing and freeze substitution. F Electron Microsc 1983; 32: 305-20.

19 Hunziker E B, Schenk R K. Cartilage ultrastructure after high pressure freezing, freeze substitution, and low temperature embedding. II. Intercellular matrix ultratemperature embedding. II. Intercellular matrix ultrastructure-preservation of proteog
state. $\mathcal{F}$ Cell Biol 1984; 98: $277-82$.

20 Aresenault A L, Ottensmeyer F P, Heath I B. An electron microscopic and spectroscopic study of murine epiphyseal cartilage: analysis of fine structure and matrix vesicles preserved by slam freezing and freeze substitution. $\mathcal{F}$ Ultrastruct Mol Struct Res 1988; 98: 32-47.

21 Keene D R, McDonald K. The ultrastructure of the connective tissue matrix of skin and cartilage after highpressured freezing and freeze-substitution. $\mathcal{F}$ Histochem Cytochem 1993; 41: 1141-53.

22 Jeremy G P, Gardner D L. Surface structure of baboon (Papio anubis) hydrated articular cartilage: study of low (Papio anubis) hydrated articular cartilage: study of low temperature replicas by transmission
microscopy. $\mathcal{F}$ Med Primatol 1987; 16: 301-9.

23 Gardner D L, O'Connor P, Middleton J F S, Oates K, Orford C R. An investigation by transmission electron
microscopy of freeze replicas of dog articular cartilage microscopy of freeze replicas of dog articular cartilage
surfaces: the fibre rich surface structure. $\mathcal{F}$ Anat 1983 ; surfaces: the

24 Gardner D L, O'Connor P, Oates K. Low temperature scanning electron microscopy of dog and guinea-pig hyaline articular cartilage. $\mathcal{F}$ Anat $1981 ; 132: 267-82$.

25 Heuser J. Preparing biological samples for stereomicroscopy by the quick-freeze, deep-etch, rotary-replication technique. Methods Cell Biol 1981;22: 97-122.

26 Mecham R P, Heuser J. Three-dimensional organization of extracellular matrix in elastic cartilage as viewed by quick
freeze, deep etch electron microscopy. Connect Tissue Res 1990; 24: 83-93.

27 Ohno S, Fujii Y Three-dimensional and histochemical studies of peroxisomes in cultured hepatocytes by quick- freezing and deep-etching method. Histochem $\mathcal{F}$ 1990; 22: $143-54$.

28 Ohno S, Fujii Y. Three-dimensional studies of the cytoskeleton of cultured hepatocytes: a quick-freezing and deep-etching study. Virchows Arch A 1991; 418: 61-70.

29 Naramoto A, Ohno S, Itoh N, et al. Ultrastructure of matriceal changes in chronic phase of Masugi nephritis by quick-freezing and deep-etching method. Virchow's Arch $A$ 1991; 418: 51-9.

30 Naramoto A, Ohno S, Nakazawa K, Takami H, Itoh $\mathrm{N}$, Shigematsu $\mathrm{H}$. Three-dimensional ultrastructure of glomerular injury in serum sickness nephritis using the quick-freezing and deep-etching method. Virchows Arch A 1991; 418: $185-92$.

31 Yoshimura A, Ohno S, Nakano K, et al. Three-dimensional ultrastructure of anionic sites of the glomerular basement membrane by a quick-freezing and deep-etching method using a cationic tracer. Histochemistry 1991; 96: 107-13.

32 Menco B M. A survey of ultra-rapid cryofixation methods with particular emphasis on applications to freezefracturing, freeze-etching, and freeze-substitution. $f$ Electron Microsc Tech 1986; 4: 177-240.

33 Paulsson $\mathrm{M}$, Heinegård $\mathrm{D}$. Purification and structural localization of a cartilage matrix protein. Biochem $\mathcal{F} 1981$; 197: 367-75.

34 Rosier de D J, Klug A. Reconstruction of three dimensional structures from electron micrographs. Nature 1968; 217: $130-4$

35 Wellaver P, Wyler T, Buddecke E. Electron microscopic and physico-chemical studies on bovine nasal cartilage proteoglycan. Hoppe-Seyler's Z Physiol Chem 1972; 353: 1043-52.

36 Rosenberg L, Hellmann W, Kleinschmidt A K. Electron microscopic studies of proteoglycans aggregates from bovine articular cartilage. $f$ Biol Chem 1975; 250: 1877-83.

37 Thyberg J, Lohmander S, Heinegàrd D. Proteoglycans of hyaline cartilage. Electron-microscopic studies on isolated molecules. Biochem ₹ 1975; 151: 157-66.

38 Heinegård $\mathrm{D}$, Lohmander S, Thyberg J. Cartilage proteoglycan aggregates. Electron-microscopic studies of native glycan aggregates. Electron-microscopic studies of native
and fragmented molecules. Biochem $\mathcal{F} 1978 ; 175: 913-9$.

39 Kimura J H, Osdoby P, Caplan A I, Hascall V C. Electron microscopic and biochemical studies of proteoglycan polydispersity in chick limb bud chondrocyte cultures. $\mathcal{F}$ Biol Chem 1978; 253: 4721-9.

40 Hascall G K. Cartilage proteoglycan: comparison of sectioned and spread whole molecules. $f$ Ultrastruct Res 1980; 70: 369-75.

41 Hardingham T. Proteoglycans: their structure, interactions and molecular organization in cartilage. Biochem Soc Trans 1981; 9: 489-97.

42 Buckwalter J A, Rosenberg L C. Electron microscopic studies of cartilage proteoglycans. Direct evidence for the variable length of the chondroitin sulfate-rich region of proteoglycan subunit core protein. F Biol Chem 1982; 257: proteoglyca

43 Wiedemann H, Paulsson M, Timpl R, Engel J, Heinegård D. Domain structure of cartilage proteoglycans visualized by rotary shadowing of intact and fragmented molecules. Biochem $\mathcal{F}$ 1984; 224: 331-3.

44 Buckwalter J A, Rosenberg L C. Electron microscopic studies of cartilage proteoglycans. Electron Microsc Rez 1988; 1: 87-112.

45 Poole A R, Pidoux I, Reiner A, Rosenberg L. An immunoelectron microscope study of the organization of proteoglycan monomer, link protein, and collagen in the
matrix of articular cartilage. $\mathcal{F}$ Cell Biol 1982; 93: 921-37.

46 Mender M, Bender S G E, Vaughan L, Winterhalter K H, Bruckner P. Cartilage contains mixed fibrils of collagen Bruckner P. Cartilage contains mixed fibrils of
type II, IX, and XI. $\mathcal{F}$ Cell Biol 1989; 108: 191-7.

47 Glauser W M, Humbel B, Glatt M, Stäuli P, Winterhalter KH, Bruckner P. On the role of type IX collagen in the extracellular matrix of cartilage: type IX collagen is localized to intersections of collagen fibrils. $\mathcal{F}$ Cell Biol 1986; 102: 1931-9

48 McCormick D, van der Rest M, Goodship J, Lozano G, Ninomiya Y, Olsen B R. Structure of the glycosaminoglycan domain in the type IX collagen-proteoglycan. Proc Natl Acad Sci USA 1987; 84: 4044-8.

49 Vaughan L, Mendler M, Huber S, et al. D-periodic distribution of collagen type IX along cartilage fibrils. $\mathcal{F}$ Cell Biol 1988; 106: 991-7.

50 van der Rest M, Mayne R. Type IX collagen proteoglycan from cartilage is covalently cross-linked to type II from cartilage is covalently cross-linked

51 Krusius T, Ruoslahti E. Primary structure of an extracellular matrix proteoglycan core protein deduced from cloned cDNA. Proc Natl Acad Sci USA 1986; 83: 7683-7.

52 Oldberg $\AA$, Antonsson $\mathrm{P}$, Lindblom $\mathrm{K}$, Heinegård D. A collagen-binding 59-kd protein (fibromodulin) is structurally related to the small interstitial proteoglycans
PG-S1 and PG-S2 (decorin). EMBO $₹ 1989 ; 8: 2601-4$.

53 Vogel K G, Paulsson M, Heinegård D. Specific inhibition of type I and type II collagen fibrogenesis by the low molecular mass proteoglycan of tendon. Biochem $\mathcal{F} 1984$; 223: $587-97$

54 Hedbom E, Heinegård D. Interaction of a $59 \mathrm{kDa}$ connective tissue matrix protein with collagen I and collagen II. $\mathcal{f}$ Biol Chem 1989; 264: 6898-905.

55 Yamaguchi Y, Mann D M, Ruoslahti E. Negative regulation of transforming growth factor- $\beta$ by the proteoglycan decorin. Nature 1990; 346: 281-4. 